\title{
The size of collision solutions in orbital elements space
}

\author{
G.B. Valsecchi ${ }^{1}$, A. Rossi ${ }^{2}$, A. Milani ${ }^{3}$ and S.R. Chesley ${ }^{4}$ \\ ${ }^{1}$ INAF-IASF, via Fosso del Cavaliere 100, 00133 Roma, Italy \\ email: giovanni@rm.iasf.cnr.it \\ ${ }^{2}$ ISTI-CNR, Area della Ricerca di Pisa, Via G. Moruzzi 1, 56124 Pisa, Italy \\ ${ }^{3}$ Department of Mathematics, University of Pisa, Via Buonarroti 2, 56127 Pisa, Italy \\ ${ }^{4}$ Jet Propulsion Laboratory, 4800 Oak Grove Drive, CA-91109 Pasadena, USA
}

\begin{abstract}
In the framework of the analytical theory of close encounters, and under suitable assumptions, we compute the size of the region in orbital elements space containing collisions solutions. In the linearized approximation in the semimajor axis/eccentricity plane the collision region is the interior of an ellipse. Examples are given from past cases of Near Earth Asteroids having the possibility of impacting our planet.
\end{abstract}

Keywords. Collisions, Near-Earth asteroids, planetary close encounters

\section{Introduction}

Impact monitoring programs like CLOMON2 and Sentry (Milani et al. 2004) routinely find collision possibilities of Near-Earth Asteroids (NEAs) with the Earth at specific dates, and characterize them with values of quantities like the stretching and the impact probability (Milani et al. 1999, Milani et al. 2004, Milani et al. 2002). These quantities depend, among other things, on the amount of observational data available for the given NEA, and on assumptions on the statistical features of the data; thus, they do not depend only on the orbital parameters of the NEA.

The question we address here is the following: leaving aside observations and statistics, and considering only the celestial mechanics side of the problem, can we meaningfully speak of the "size" of a collision solution and, if yes, how does it vary for different NEA collisions? We look for an answer in the framework of the recent extension (Valsecchi et al. 2003, Valsecchi 2004) of the analytical theory of close encounters (Öpik 1976).

\section{Collision solutions}

Let us start by considering the points in the space of orbital elements leading to an exact collision with a point-mass Earth. We consider the Earth to be on a circular orbit of radius $1 \mathrm{AU}$. The conditions on the orbital elements of the NEA for an exact collision at a given time $t$ are:

$$
\begin{aligned}
\frac{a\left(1-e^{2}\right)}{1 \pm e \cos \omega} & =1 \\
\Omega-\lambda_{\oplus} & =\frac{\pi}{2} \mp \frac{\pi}{2} \\
\omega+f & =\frac{\pi}{2} \mp \frac{\pi}{2},
\end{aligned}
$$


where the upper sign is for collisions at the ascending node and $\lambda_{\oplus}$ is the longitude of the Earth at the time $t$. Thus the collision solutions for a given date $t$ lie on a 3 -dimensional manifold in the 6-dimensional space of orbital elements.

Note that for a collision at another date equations (2.1)-(2.3) would be the same, with only $\lambda_{\oplus}$ changed. Thus, if we look for the set of collision solutions at an arbitrary date, equation (2.2) would not introduce any constraint on the orbital elements $(a, e, i, \omega, f)$, since $\Omega$ can be fixed at the suitable value as a function of $\lambda_{\oplus}$.

In the $b$-plane of Öpik's theory (Valsecchi et al. 2003), the exact collision condition is the point of coordinates $\xi=\zeta=0$. The impact radius of the Earth on that plane is:

$$
b_{\oplus}=\sqrt{r_{\oplus}^{2}+2 c r_{\oplus}}
$$

where $c=m / U^{2}, m$ is the ratio of the mass of the Earth to that of the Sun,

$$
U=\sqrt{3-\frac{1}{a}-2 \sqrt{a\left(1-e^{2}\right)} \cos i}
$$

is the unperturbed geocentric speed of the NEA in units of the heliocentric velocity of the Earth, and $r_{\oplus}$ is the actual radius of the Earth; the unit of length used here is the AU. In the following, we use $b_{\oplus}$ to compute the region in elements space corresponding to a physical collision.

\subsection{The correspondence between orbital elements and b-plane coordinates}

If at $t=t_{0}$ the small body is near the Earth, at the point of geocentric cartesian coordinates $\left(X_{0}, Y_{0}, Z_{0}\right)$, we have

$$
\begin{aligned}
& \xi=X_{0} \cos \phi-Z_{0} \sin \phi \\
& \zeta=\left(X_{0} \sin \phi+Z_{0} \cos \phi\right) \cos \theta-Y_{0} \sin \theta,
\end{aligned}
$$

where $\theta=\theta(a, e, i)$ and $\phi=\phi(a, e, i)$ are the usual angles of Öpik's theory, given by:

$$
\begin{aligned}
& \cos \theta=\frac{\sqrt{a\left(1-e^{2}\right)} \cos i-1}{\sqrt{3-\frac{1}{a}-2 \sqrt{a\left(1-e^{2}\right)} \cos i}} \\
& \sin \theta=\frac{\sqrt{2-\frac{1}{a}-a\left(1-e^{2}\right) \cos ^{2} i}}{\sqrt{3-\frac{1}{a}-2 \sqrt{a\left(1-e^{2}\right)} \cos i}} \\
& \sin \phi= \pm \frac{\sqrt{2-\frac{1}{a}-a\left(1-e^{2}\right)}}{\sqrt{2-\frac{1}{a}-a\left(1-e^{2}\right) \cos ^{2} i}} \\
& \cos \phi= \pm \frac{\sqrt{a\left(1-e^{2}\right)} \sin i}{\sqrt{2-\frac{1}{a}-a\left(1-e^{2}\right) \cos ^{2} i}} .
\end{aligned}
$$

In the expression for $\sin \phi$ the upper sign applies to encounters in the post-perihelion branch of the orbit, and in that for $\cos \phi$ to encounters close to the ascending node.

By using equations (2.1)-(2.3) we can express $X_{0}, Y_{0}, Z_{0}$ as functions of the orbital parameters (to first order in $X_{0}, Y_{0}, Z_{0}$ ):

$$
\begin{aligned}
& X_{0}=\frac{a\left(1-e^{2}\right)}{1+e \cos f_{0}}-1 \\
& Y_{0}=\Omega+\frac{\pi}{2} \mp\left\{\frac{\pi}{2}-\arctan \left[\cos i \tan \left(\omega+f_{0}\right)\right]\right\}-\lambda_{\oplus}
\end{aligned}
$$




$$
Z_{0}=\sin i \sin \left(\omega+f_{0}\right)
$$

where the upper sign is for collisions at the ascending node.

Let us now consider a NEA that, at a generic epoch $t^{*}$, has orbital parameters $a, e$, $i, \omega, \Omega, f$, and at $t=t_{0}$, when its true anomaly is $f_{0}$, has a collision with the Earth, located (at $t=t_{0}$ ) at longitude $\lambda_{\oplus}$. Note that

$$
t_{0}=t^{*}+2 h \pi a^{3 / 2}
$$

where $h$ is the non-integer number of heliocentric revolutions made by the NEA between $t^{*}$ and $t_{0}$.

To understand what happens to $\xi$ and $\zeta$, when we apply small changes to the orbital elements, we can compute

$$
\begin{aligned}
d \xi & =\sum_{i=1,6} \frac{\partial \xi}{\partial \mathcal{E}_{i}} d \mathcal{E}_{i} \\
d \zeta & =\sum_{i=1,6} \frac{\partial \zeta}{\partial \mathcal{E}_{i}} d \mathcal{E}_{i},
\end{aligned}
$$

where $\mathcal{E}_{1}=a, \mathcal{E}_{2}=e, \mathcal{E}_{3}=i, \mathcal{E}_{4}=\omega, \mathcal{E}_{5}=\Omega, \mathcal{E}_{6}=f$.

\subsection{The derivatives of $\xi, \zeta$ with respect to the elements}

At the collision, the derivatives $\partial \xi / \partial \mathcal{E}_{i}, \partial \zeta / \partial \mathcal{E}_{i}$ have the form:

$$
\begin{aligned}
\frac{\partial \xi}{\partial \mathcal{E}_{i}} & =\frac{\partial X_{0}}{\partial \mathcal{E}_{i}} \cos \phi-\frac{\partial Z_{0}}{\partial \mathcal{E}_{i}} \sin \phi \\
\frac{\partial \zeta}{\partial \mathcal{E}_{i}} & =\frac{\partial X_{0}}{\partial \mathcal{E}_{i}} \cos \theta \sin \phi+\frac{\partial Z_{0}}{\partial \mathcal{E}_{i}} \cos \theta \cos \phi-\frac{\partial Y_{0}}{\partial \mathcal{E}_{i}} \sin \theta
\end{aligned}
$$

where we have dropped the terms with either $X_{0}$, or $Y_{0}$, or $Z_{0}$ as factor (since $X_{0}=$ $\left.Y_{0}=Z_{0}=0\right)$. Note that, among the derivatives, only $\partial \xi / \partial a$ and $\partial \zeta / \partial a$ depend on the elapsed time $t_{0}-t^{*}$; their expressions contain $h$, the non-integer number of heliocentric revolutions made by the NEA between $t^{*}$ and $t_{0}$.

We can then write:

$$
\left(\begin{array}{l}
\delta \xi \\
\delta \zeta
\end{array}\right)=\left(\begin{array}{llllll}
\frac{\partial \xi}{\partial a} & \frac{\partial \xi}{\partial e} & \frac{\partial \xi}{\partial i} & \frac{\partial \xi}{\partial \omega} & \frac{\partial \xi}{\partial \Omega} & \frac{\partial \xi}{\partial f} \\
\frac{\partial \zeta}{\partial a} & \frac{\partial \zeta}{\partial e} & \frac{\partial \zeta}{\partial i} & \frac{\partial \zeta}{\partial \omega} & \frac{\partial \zeta}{\partial \Omega} & \frac{\partial \zeta}{\partial f}
\end{array}\right)\left(\begin{array}{c}
\delta a \\
\delta e \\
\delta i \\
\delta \omega \\
\delta \Omega \\
\delta f
\end{array}\right)
$$

Actually, since an explicit computation shows that $\partial \xi / \partial i=\partial \xi / \partial \Omega=\partial \zeta / \partial i=0$, we have that:

$$
\left(\begin{array}{c}
\delta \xi \\
\delta \zeta
\end{array}\right)=\left(\begin{array}{ccccc}
\frac{\partial \xi}{\partial a} & \frac{\partial \xi}{\partial e} & \frac{\partial \xi}{\partial \omega} & 0 & \frac{\partial \xi}{\partial f} \\
\frac{\partial \zeta}{\partial a} & \frac{\partial \zeta}{\partial e} & \frac{\partial \zeta}{\partial \omega} & \frac{\partial \zeta}{\partial \Omega} & \frac{\partial \zeta}{\partial f}
\end{array}\right)\left(\begin{array}{c}
\delta a \\
\delta e \\
\delta \omega \\
\delta \Omega \\
\delta f
\end{array}\right) .
$$

Note that, to first order, the changes in $(\xi, \zeta)$ do not depend upon the inclination $i$; however, the partial derivatives do depend upon $i$, as also evidenced by the examples given in the next Section. 


\section{Collision solution size in $a, e$}

As we have seen, the 2-dimensional vector giving a small displacement on the $b$-plane as function of the orbital elements is given by the product of a 2 rows by 6 columns matrix times a 6 -dimensional vector in elements space (see equation 2.4). This corresponds to the fact that each point on the $b$-plane, if the time of close approach is variable, has as preimage a 4-dimensional manifold. However, if we fix four elements and leave only two as variables, the inversion becomes possible. To select the two elements we want to use as parameters, we have to take into account that the coordinates $(i, \Omega)$ play a different role: the partial derivatives with respect to $i$ are zero, and $\Omega$ is only a function of $\lambda_{\oplus}$, that is of the close approach time $t_{0}$.

If, for example, we set $\delta \omega=\delta \Omega=\delta f=\delta i=0$, we can find the size of a collision solution in the $a-e$ plane. In this case, in fact:

$$
\left(\begin{array}{l}
\delta \xi \\
\delta \zeta
\end{array}\right)=\left(\begin{array}{ll}
\frac{\partial \xi}{\partial a} & \frac{\partial \xi}{\partial e} \\
\frac{\partial \zeta}{\partial a} & \frac{\partial \zeta}{\partial e}
\end{array}\right)\left(\begin{array}{l}
\delta a \\
\delta e
\end{array}\right)
$$

this can be inverted to give:

$$
\left(\begin{array}{l}
\delta a \\
\delta e
\end{array}\right)=\frac{1}{\left|\begin{array}{ll}
\frac{\partial \xi}{\partial a} & \frac{\partial \xi}{\partial e} \\
\frac{\partial \zeta}{\partial a} & \frac{\partial \zeta}{\partial e}
\end{array}\right|}\left(\begin{array}{cc}
\frac{\partial \zeta}{\partial e} & -\frac{\partial \xi}{\partial e} \\
-\frac{\partial \zeta}{\partial a} & \frac{\partial \xi}{\partial a}
\end{array}\right)\left(\begin{array}{l}
\delta \xi \\
\delta \zeta
\end{array}\right) .
$$

Using the above expressions we can find the values of $\delta a$ and $\delta e$ corresponding to a circle of radius $b_{\oplus}$ in the $b$-plane. The shape of the collision region, in this linear approximation, is an ellipse; it does not need to have the major and minor axes aligned with the coordinate axes.

\subsection{Application: the 2019 collision of $2002 \mathrm{NT}_{7}$ and the 2008 collision of $2003 \mathrm{EE}_{16}$}

We now compute the sizes in the $a-e$ plane of the collision solutions of two NEAs that have been of some interest in the recent past.

In the summer of 2002 the confidence region of $2002 \mathrm{NT}_{7}$ in orbital elements space contained a collision taking place about $h=7.3$ revolutions of the asteroid later, in early 2019. The value of the Palermo scale (Chesley et al. 2002) for this collision became, for a short time, larger than 0 , before coming down quickly with the accumulation of new observations.

Substituting the numeric values of the orbital elements of $2002 \mathrm{NT}_{7}(a=1.74, e=0.53$, $i=42.3)$ in the expressions for $\delta a$ and $\delta e$ we get:

$$
\begin{aligned}
\delta a & =\frac{-0.19 \delta \xi-1.7 \delta \zeta}{3.4 h} \\
\delta e & =\frac{0.09 \delta \xi+0.79 \delta \zeta}{-4.9 h}-0.62 \delta \xi .
\end{aligned}
$$

The left panel of Fig. 1 shows this collision in the $a$-e plane; to highlight the dependence on $h$ (the non-integer number of heliocentric revolutions made by $2002 \mathrm{NT}_{7}$ before the collision), in the right panel we show the size of two collisions characterized by $h=1$ and $h=50$. The shrinking of the region along $a$, as $h$ grows, is quite noticeable, as is the change in the overall slope of the collision region.

In March 2003 the confidence region of $2003 \mathrm{EE}_{16}$ in orbital elements space contained a collision taking place about $h=2.9$ revolutions of the asteroid later, in early 2008 . This collision solution did not go above the 0 of the Palermo scale at any time. Proceeding as 

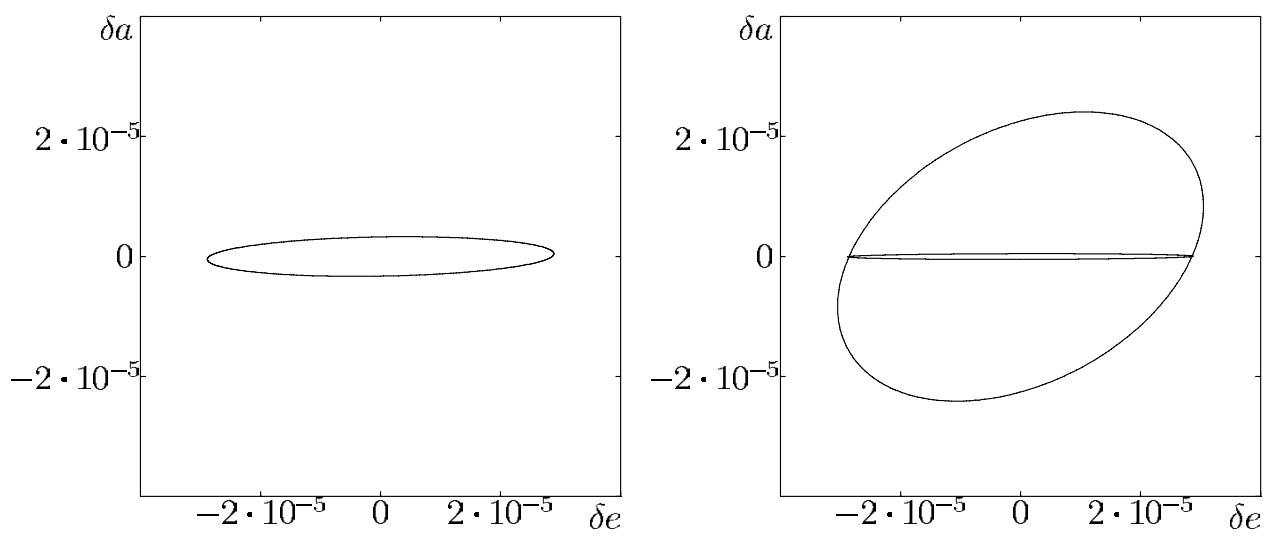

Figure 1. Left: the size of the collision solution of $2002 \mathrm{NT}_{7}$ in $\delta e, \delta a$ space for $h=7.3$. Right: the size of collision solutions, of the same NEA, for $h=1$ (larger ellipse) and $h=50$.
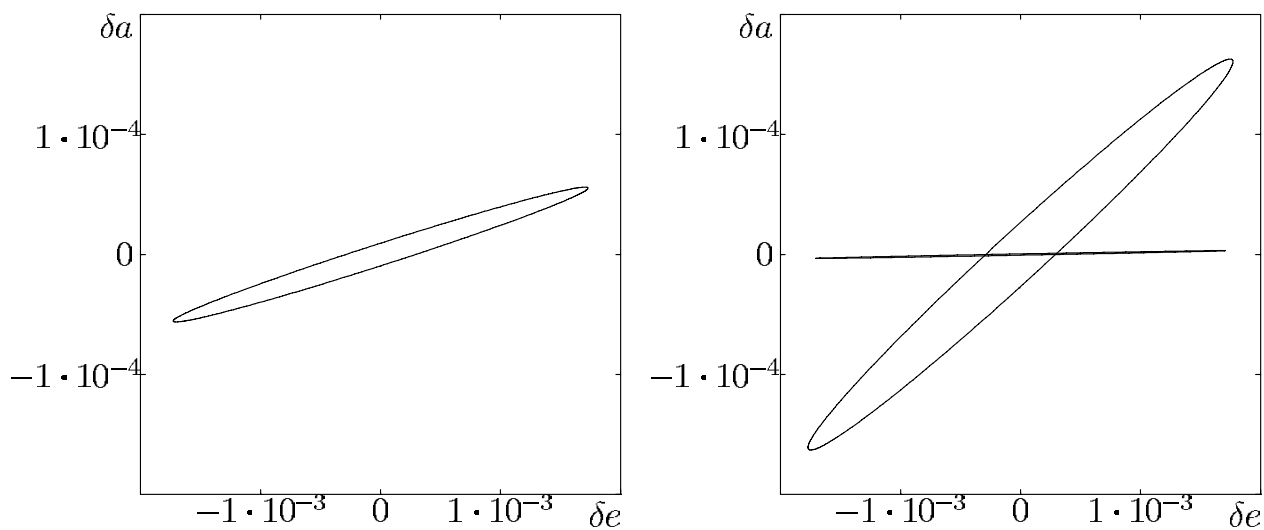

Figure 2. Left: the size of the collision solution of $2003 \mathrm{EE}_{16}$ in $\delta e, \delta a$ space for $h=2.9$. Right: the size of collision solutions, of the same NEA, for $h=1$ (larger ellipse) and $h=50$. Note that the scale for $\delta a$ is much smaller than for $\delta e$, that is, the ellipse is much wider in the $\delta e$ direction.

before, for $a=1.42, e=0.62, i=0.6$ (the orbital elements of $2003 \mathrm{EE}_{16}$ ), we get:

$$
\begin{aligned}
\delta a & =\frac{-0.19 \delta \xi-0.03 \delta \zeta}{0.059 h} \\
\delta e & =\frac{0.09 \delta \xi+0.02 \delta \zeta}{-0.071 h}-35 \delta \xi .
\end{aligned}
$$

Figure 2 shows the same information as Fig. 1 for $2003 \mathrm{EE}_{16}$. The most important difference between the two cases is the scale of the Figures: the collision solution of $2003 \mathrm{EE}_{16}$ is more than an order of magnitude larger in both coordinates. Of course this is due to the much lower inclination of $2003 \mathrm{EE}_{16}$.

\section{Conclusions}

We have shown that, under suitable assumptions, it is possible to deduce analytical expressions giving the size of the region in orbital elements space containing orbits colliding with the Earth at a given date. When computed in the $a-e$ plane, the size depends on the number of heliocentric revolutions $h$ made by the NEA before hitting the Earth, and is smaller for larger $h$. 
Applying the above mentioned expressions to two NEAs, we find that the size of the collision region in the $a$-e plane differs significantly in the two cases.

We plan to continue this research, with the goal of providing an easy to compute first approximation of the location of a Virtual Impactor. This could be a useful tool for the impact monitoring systems.

\section{Acknowledgements}

We are grateful to G.F. Gronchi and G. Tommei for useful discussions on the subject of this paper.

\section{References}

Chesley, S.R., Chodas, P.W., Milani, A., Valsecchi, G.B., and Yeomans, D.K. 2002, Icarus 159, 423

Milani, A., Chesley, S.R., and Valsecchi, G.B. 1999, Astron. Astrophys. 346, L65

Milani, A., Chesley, S.R., and Valsecchi, G.B. 2000, Planet. ES Space Sci. 48, 945

Milani, A., Chesley, S.R., Chodas, P.W., and Valsecchi, G.B. 2002, in: W.F. Bottke Jr., A. Cellino, P. Paolicchi and R.P. Binzel (eds.), Asteroids III, Univ. Arizona Press, p. 55

Milani, A., Chesley, S.R., Sansaturio, M.E., Tommei, G. and Valsecchi, G.B. 2004, Icarus, in press

Opik, E.J. 1976, Interplanetary Encounters, Elsevier

Valsecchi, G.B., Milani, A., Gronchi, G.-F., and Chesley, S.R. 2003, Astron. Astrophys. 408, 1179

Valsecchi, G.B. 2004, in: J. Souchay, S. Thoms, and G.Hakuba (eds.), Dynamics of non point-like celestial bodies and of the rings, Springer, in press 